\title{
Existence of solutions for a class of second-order impulsive Hamiltonian system with indefinite linear part
}

\section{Qiongfen Zhang}

College of Science, Guilin University of Technology, Guilin, Guangxi 541004, P. R. China.

Communicated by M. Bohner

\begin{abstract}
We consider a class of second-order impulsive Hamiltonian system with indefinite linear part. By using saddle point theorem in critical point theory, an existence result is obtained, which extends and improves some existing results.
\end{abstract}

Keywords: Impulsive Hamiltonian system, saddle point theorem, solutions, existence.

2010 MSC: 39A11, 58E05, 70H05.

(C)2018 All rights reserved.

\section{Introduction}

Consider the following second-order impulsive Hamiltonian system with indefinite linear part

$$
\left\{\begin{array}{l}
\ddot{u}(t)-A(t) u(t)=-\nabla F(t, u(t)), t \in[0,1] \backslash\left\{t_{1}, t_{2}, \cdots, t_{m}\right\}, \\
u(0)=u(1)=0, u\left(t_{i}^{+}\right)=u\left(t_{i}^{-}\right), i=1, \cdots, m, \\
\dot{u}\left(t_{i}^{+}\right)-\dot{u}\left(t_{i}^{-}\right)=I_{i}\left(u\left(t_{i}\right)\right), i=1, \cdots, m,
\end{array}\right.
$$

where $A \in C\left([0,1], \mathbb{R}^{N \times N}\right)$ is a continuous map with values in the space of $N \times N$ symmetric matrices, $u \in \mathbb{R}^{N}, F:(0,1) \times \mathbb{R}^{N} \rightarrow \mathbb{R}$ is a Carathéodory function, $I_{i}$ are continuous functions on $\mathbb{R}^{N}$, and

$$
u\left(t_{i}^{\mp}\right)=\lim _{t \rightarrow t_{i}^{\mp}} u(t), \quad \dot{u}\left(t_{i}^{\mp}\right)=\lim _{t \rightarrow t_{i}^{\mp}} \dot{u}(t) .
$$

When $A(t)=0$ and $u(t)$ is defined in $(0,1)$ with values in $\mathbb{R},(1.1)$ reduced to the following impulsive problems

$$
\left\{\begin{array}{l}
\ddot{u}(t)=-\nabla F(t, u(t)), \quad t \in[0,1] \backslash\left\{t_{1}, t_{2}, \cdots, t_{m}\right\}, \\
u(0)=u(1)=0, u\left(t_{i}^{+}\right)=u\left(t_{i}^{-}\right), i=1, \cdots, m, \\
\dot{u}\left(t_{i}^{+}\right)-\dot{u}\left(t_{i}^{-}\right)=I_{i}\left(u\left(t_{i}\right)\right), i=1, \cdots, m .
\end{array}\right.
$$

Email address: qfzhangcsu@163.com (Qiongfen Zhang)

doi: $10.22436 /$ jnsa.011.03.05

Received: 2017-08-15 Revised: 2017-12-27 Accepted: 2018-01-11 
Impulsive problems arise naturally in real world. As we know, many mathematical models involve impulses. Since impulsive problems are important in real world, many researchers have extensively studied the theory and applications of impulsive differential equations, see $[2,4,5,8,11]$ for more details. In recent years, variational methods have been widely used to study Hamiltonian system and impulsive problems, see [1, 3, 6, 7, 9, 12-17] and references therein. In [7], Kyritsi and Papageorgion investigated problem (1.2) and obtained an existence result by using Morse critical groups. In [1], Agarwal, Bhaskar and Perea studied problem (1.2)) via Morse theory and got some results. Later, Wang and Wang [13] obtained an existence result for problem (1.2) under some conditions on $\nabla \mathrm{F}(\mathrm{t}, \mathrm{u}(\mathrm{t}))$ by using saddle point theorem, which extends and improves the results in [1] and [7].

Motivated mainly by $[1,7,13]$, we consider problem (1.1) with indefinite linear part by saddle point theorem and obtain an existence result, which seems not to be considered previously. For convenience, $C_{i}$ denote different positive constants in the following.

Suppose the following hypotheses hold:

(H1) $\nabla \mathrm{F}(\mathrm{t}, \mathrm{u})$ is asymptotically piecewise linear and

$$
\nabla F(t, u)=\sum_{i=1}^{m+1} a_{i} \chi_{i}(t) u+g(t, u),
$$

where $a_{1}, \ldots, a_{m+1} \in \mathbb{R}$,

$$
x_{i}(t)= \begin{cases}1, & t \in\left(t_{i-1}, t_{i}\right) \\ 0, & t \in(0,1) \backslash\left(t_{i-1}, t_{i}\right)\end{cases}
$$

is the characteristic function of the subinterval $\left(t_{i-1}, t_{i}\right)$ and there are $r \in(1,2)$ and two positive constants $\mathrm{C}_{1}$ and $\mathrm{C}_{2}$ such that

$$
|g(t, u)| \leqslant C_{1}|u|^{r-1}+C_{2} \text { for a.e. } t \in[0,1] \text { and all } u \in \mathbb{R}^{N} \text {. }
$$

(H2) There are positive constants $\mathrm{C}_{3}, \mathrm{C}_{4}$ and $\mu>2$ such that

$$
\left(u, I_{i}(u)\right) \geqslant C_{3}|u|^{\mu}-C_{4}, \forall u \in \mathbb{R}^{N}, i=1,2, \ldots, m .
$$

We only consider the nonresonant case, where for all $i \in\{1, \cdots, m+1\}, a_{i}$ is not in the set

$$
\Sigma_{i}=\left\{\lambda_{k}^{i}: 0 \leqslant \lambda_{1}^{i} \leqslant \lambda_{2}^{i} \leqslant \cdots \leqslant \lambda_{k}^{i} \rightarrow+\infty, k=1,2, \ldots\right\}
$$

of the eigenvalues of the problem

$$
\left\{\begin{array}{l}
-\ddot{u}(t)+A(t) u(t)=\lambda u(t), t \in\left(t_{i-1}, t_{i}\right), \\
u\left(t_{i-1}\right)=u\left(t_{i}\right)=0, i=1, \cdots, m+1,
\end{array}\right.
$$

The main result is the following.

Theorem 1.1. Assume that (H1) and (H2) hold, and $\mathrm{a}_{i} \notin \Sigma_{i}$ for $i=1,2, \ldots, m+1$, then problem (1.1) has one nontrivial homoclinic solution.

\section{Preliminaries}

Let $W$ be the Sobolev space defined by

$$
W=W_{\text {per }}^{1,2}\left((0,1), \mathbb{R}^{N}\right)=\left\{u \in W^{1,2}\left((0,1), \mathbb{R}^{N}\right): u(0)=u(1)\right\}
$$

with the inner product

$$
(\mathrm{u}, v)=\int_{0}^{1}[(\dot{\mathrm{u}}(\mathrm{t}), \dot{v}(\mathrm{t}))+(\mathrm{A}(\mathrm{t}) \mathrm{u}(\mathrm{t}), v(\mathrm{t}))] \mathrm{dt} .
$$

The (compact) embedding of $W^{1,2}\left((0,1), \mathbb{R}^{N}\right)$ into $C\left([0,1], \mathbb{R}^{N}\right)$ shows that this definition makes sense. It 
is easy to see that weak solutions of problem (1.1) coincide with the critical points of the $\mathrm{C}^{1}$-functional

$$
\varphi(u)=\frac{1}{2} \int_{0}^{1}\left[|\dot{u}(t)|^{2}+(A(t) u(t), u(t))\right] d t-\int_{0}^{1} F(t, u(t)) d t-\sum_{i=1}^{m} \hat{I}_{i}\left(u\left(t_{i}\right)\right), u \in W,
$$

where $\hat{I}_{\mathfrak{i}}\left(\mathfrak{u}\left(\mathfrak{t}_{\mathfrak{i}}\right)\right)=\int_{0}^{\mathfrak{u}\left(\mathfrak{t}_{\mathfrak{i}}\right)} I(s)$ ds. Since every $\hat{I}_{\mathfrak{i}}(0)=0$ for $\mathfrak{i}=1,2, \ldots, m$, the closed linear subspace

$$
\mathcal{N}=\left\{u \in W: u\left(t_{i}\right)=0, i=1,2, \ldots, m\right\}
$$

is important here. For $i=1,2, \ldots, m$, the mapping $W \mapsto \mathbb{R}^{N}, u \mapsto u\left(t_{i}\right)$ is a bounded linear functional on $W$ and hence there is a unique $w_{i}$ such that $u\left(t_{i}\right)=\left(u, w_{i}\right)$ by the Riesez-Frechet representation theorem. In fact,

$$
w_{i}(t)= \begin{cases}\left(1-t_{i}\right) t, & t \in\left[0, t_{i}\right] \\ t_{i}(1-t), & t \in\left[t_{i}, 1\right] .\end{cases}
$$

Since $t_{i}$ are distinct, $\omega_{i}$ are linearly independent, so $\mathcal{N}$ is the orthogonal complement of the $m$-dimensional subspace $\mathcal{M}$ that they span. Hence we have the orthogonal decomposition

$$
\mathrm{W}=\mathcal{N} \bigoplus \mathcal{M}, \mathrm{u}=v+w,
$$

and

$$
\begin{aligned}
\varphi(u)= & \frac{1}{2} \int_{0}^{1}\left[|\dot{v}(t)|^{2}+(A(t) v(t), v(t))\right] d t+\frac{1}{2} \int_{0}^{1}\left[|\dot{w}(t)|^{2}+(A(t) w(t), w(t))\right] d t \\
& -\int_{0}^{1} F(t, u(t)) d t-\sum_{i=1}^{m} \hat{I}_{i}\left(u\left(t_{i}\right)\right), u \in W .
\end{aligned}
$$

By (2.1), each $w \in \mathcal{M}$ is affine on the subintervals $\left[t_{i-1}, t_{i}\right]$. Since the space of continuous functions on $[0,1]$ that are affine on these subintervals and vanish at the endpoints is also m-dimensional, hence $\mathcal{N}$ is precisely this space. Then we also have

$$
\max _{t \in[0,1]}|w(t)|=\max _{i=1, \ldots, m}\left|w\left(t_{i}\right)\right|, \quad \forall w \in \mathcal{M},
$$

and this is an equivalent norm on this finite dimensional space.

The subspace $\mathcal{N}$ has the decomposition

$$
\mathcal{N}=\bigoplus_{i=1}^{m+1} \mathcal{N}_{i}, \quad v=\sum_{i=1}^{m+1} v_{i}
$$

where $\mathcal{N}_{i}=W^{1,2}\left(\left(t_{i-1}, t_{i}\right), \mathbb{R}^{N}\right), v_{i}=\chi_{i} v$. Combining this with (2.2) gives

$$
\begin{aligned}
\varphi(u) & =\frac{1}{2}\left[\sum_{i=1}^{m+1} \int_{t_{i-1}}^{t_{i}}\left(|\dot{v}(t)|^{2}+(A(t) v(t), v(t))\right) d t\right]+\frac{1}{2} \int_{0}^{1}\left[|\dot{w}(t)|^{2}+(A(t) w(t), w(t))\right] d t \\
& -\int_{0}^{1} F(t, u(t)) d t-\sum_{i=1}^{m} \hat{I}_{i}\left(u\left(t_{i}\right)\right) \\
& =\frac{1}{2}\|v\|^{2}+\frac{1}{2}\|w\|^{2}-\int_{0}^{1} F(t, u(t)) d t-\sum_{i=1}^{m} \hat{I}_{i}\left(u\left(t_{i}\right)\right), u \in W .
\end{aligned}
$$

The following lemma is saddle point theorem, which comes from [10] and is useful in the proof of our theorem.

Lemma 2.1 ([10]). Let $\mathrm{E}=\mathrm{V} \bigoplus \mathrm{X}$, where $\mathrm{E}$ is a real Banach space and $\mathrm{V} \neq\{0\}$ is finite dimensional. Suppose $I \in C^{1}(E, \mathbb{R})$, satisfies (PS)-condition and

(i) there is a constant $\alpha>0$ and a bounded neighborhood $\mathrm{B}$ of 0 in $\mathrm{V}$ such that $\mathrm{I}_{\partial \mathrm{B}} \leqslant \alpha$, and

(ii) there is a constant $\beta>\alpha$ such that $\left.\mathrm{I}\right|_{X} \geqslant \beta$. 
Then I possesses a critical value $\mathrm{c} \geqslant \beta$. Moreover $\mathrm{c}$ can be characterized as

$$
c=\inf _{h \in \Gamma} \max _{u \in \bar{B}} I(h(u)),
$$

where $\Gamma=\{\mathrm{h} \in \mathrm{C}(\overline{\mathrm{B}}, \mathrm{E}) \mid \mathrm{h}=\mathrm{id}$ on $\partial \mathrm{B}\}$.

Recall that I satisfies (PS)-condition if every sequence $\left\{u_{n}\right\}$ in $W$ such that $I\left(u_{n}\right)$ is bounded and $\mathrm{I}^{\prime}\left(\mathrm{u}_{\mathrm{n}}\right) \rightarrow 0$ as $\mathrm{n} \rightarrow \infty$ has a convergent subsequence.

\section{Proof of the main theorem}

Proof of Theorem 1.1. Firstly, we prove that the functional $\varphi$ satisfies (PS)-condition. Let $\left\{u_{n}\right\} \subset W$ satisfying $\varphi^{\prime}\left(u_{n}\right) \rightarrow 0$ as $n \rightarrow \infty$ and $\varphi\left(u_{n}\right)$ is bounded. We will show that $\left\{u_{n}\right\}$ has a convergent subsequence. From $(\mathrm{H} 1)$, we have

$$
F(t, u)=\sum_{i=1}^{m+1} \frac{1}{2} a_{i} x_{i}(t)|u|^{2}+G(t, u),
$$

where $G(t, u)=\int_{0}^{u} g(t, s) d s$ satisfies

$$
|G(t, u)| \leqslant C_{5}|u|^{r}+C_{6} \text { for a.e. } t \in(0,1) \text { and all } u \in \mathbb{R}^{N},
$$

where $C_{5}$ and $C_{6}$ are positive constants. From (2.3) and (3.1), we have

$$
\begin{aligned}
\varphi(u)= & \frac{1}{2}\left[\sum_{i=1}^{m+1} \int_{t_{i-1}}^{t_{i}}\left(\left|\dot{v}_{i}(t)\right|^{2}-a_{i}\left|v_{i}(t)\right|^{2}\right) d t+\int_{0}^{1}|\dot{w}(t)|^{2} d t-\sum_{i=1}^{m+1} a_{i} \int_{t_{i-1}}^{t_{i}}|w(t)|^{2} d t\right] \\
& -\sum_{i=1}^{m+1} a_{i} \int_{t_{i-1}}^{t_{i}}\left(v_{i}(t), w(t)\right) d t+\frac{1}{2} \int_{0}^{1}(A(t) w(t), w(t)) d t \\
& +\frac{1}{2} \sum_{i=1}^{m+1} \int_{t_{i-1}}^{t_{i}}\left(A(t) v_{i}(t), v_{i}(t)\right) d t-\int_{0}^{1} G(t, u(t)) d t-\sum_{i=1}^{m} \hat{I}_{i}\left(w\left(t_{i}\right)\right), u \in W .
\end{aligned}
$$

From (H2), there exist two positive constants $C_{7}$ and $C_{8}$ such that

$$
\hat{\mathrm{I}}_{\mathrm{i}}(\mathrm{u}) \geqslant \mathrm{C}_{7}|\mathrm{u}|^{\mu}-\mathrm{C}_{8}, \quad \forall \mathrm{u} \in \mathbb{R}^{\mathrm{N}}, \mathfrak{i}=1,2, \ldots, \mathrm{m} .
$$

Let $J_{0}$ be the set of those $i$ for which $a_{i}<\lambda_{1}^{i}$ and let $J_{1}=\{1, \ldots, m+1\} \backslash J_{0}$. For each $i \in J_{1}, \lambda_{n_{i}}^{i}<a_{i}<\lambda_{n_{i}+1}^{i}$ for some $n_{i} \geqslant 1$, and we have the decomposition

$$
\mathcal{N}_{i}=\mathcal{N}_{i}^{+} \bigoplus \mathcal{N}_{i}^{-}, v_{i}=v_{i}^{+}+v_{i}^{-},
$$

where $\mathcal{N}_{i}^{-}$is the $n_{i}$-dimensional subspace spanned by the eigenfunctions of $\lambda_{1}^{i}, \ldots, \lambda_{n_{i}}^{i}$ and $\mathcal{N}_{i}^{+}$is its orthogonal complement. Then, we have

$$
\begin{aligned}
\varphi(u)= & \frac{1}{2}\left[\sum_{i \in J_{0}} \int_{t_{i-1}}^{t_{i}}\left(\left|\dot{v}_{i}(t)\right|^{2}-a_{i}\left|v_{i}(t)\right|^{2}\right) d t+\sum_{i \in J_{1}} \int_{t_{i-1}}^{t_{i}}\left(\left|\dot{v}_{i}^{+}(t)\right|^{2}-a_{i}\left|v_{i}^{+}(t)\right|^{2}\right) d t\right. \\
& \left.+\sum_{i \in J_{1}} \int_{t_{i-1}}^{t_{i}}\left(\left|\dot{v}_{i}^{-}(t)\right|^{2}-a_{i}\left|v_{i}^{-}(t)\right|^{2}\right) d t+\int_{0}^{1}|\dot{w}(t)|^{2} d t-\sum_{i=1}^{m+1} a_{i} \int_{t_{i-1}}^{t_{i}}|w(t)|^{2} d t\right] \\
& -\sum_{i=1}^{m+1} a_{i} \int_{t_{i-1}}^{t_{i}}\left(v_{i}(t), w(t)\right) d t+\frac{1}{2} \int_{0}^{1}(A(t) w(t), w(t)) d t
\end{aligned}
$$




$$
\begin{aligned}
& +\frac{1}{2} \sum_{i \in J_{0}} \int_{t_{i-1}}^{t_{i}}\left(A(t) v_{i}(t), v_{i}(t)\right) d t+\frac{1}{2} \sum_{i \in J_{1}} \int_{t_{i-1}}^{t_{i}}\left(A(t) v_{i}^{+}(t), v_{i}^{+}(t)\right) d t \\
& +\frac{1}{2} \sum_{i \in J_{1}} \int_{t_{i-1}}^{t_{i}}\left(A(t) v_{i}^{-}(t), v_{i}^{-}(t)\right) d t-\int_{0}^{1} G(t, u(t)) d t-\sum_{i=1}^{m} \hat{I}_{i}\left(w\left(t_{i}\right)\right),
\end{aligned}
$$

for

$$
u=\sum_{i \in J_{0}} v_{i}+\sum_{i \in J_{1}}\left(v_{i}^{+}+v_{i}^{-}\right)+w \in \bigoplus_{i \in J_{0}} \mathcal{N}_{i} \oplus \bigoplus_{i \in J_{1}}\left(\mathcal{N}_{i}^{+} \oplus \mathcal{N}_{i}^{-}\right) \oplus \mathcal{M}
$$

We have

$$
\begin{aligned}
& \int_{t_{i-1}}^{t_{i}}\left(\left|\dot{v}_{i}(t)\right|^{2}+\left(A(t) v_{i}(t), v_{i}(t)\right)\right) d t \geqslant \lambda_{1}^{i} \int_{t_{i-1}}^{t_{i}}\left|v_{i}(t)\right|^{2} d t, i \in J_{0}, \\
& \int_{t_{i-1}}^{t_{i}}\left(\left|\dot{v}_{i}^{+}(t)\right|^{2}+\left(A(t) v_{i}^{+}(t), v_{i}^{+}(t)\right)\right) d t \geqslant \lambda_{n_{i}+1}^{i} \int_{t_{i-1}}^{t_{i}}\left|v_{i}^{+}(t)\right|^{2} d t, i \in J_{1}, \\
& \int_{t_{i-1}}^{t_{i}}\left(\left|\dot{v}_{i}^{-}(t)\right|^{2}+\left(A(t) v_{i}^{-}(t), v_{i}^{-}(t)\right)\right) d t \leqslant \lambda_{n_{i}}^{i} \int_{t_{i-1}}^{t_{i}}\left|v_{i}^{-}(t)\right|^{2} d t, i \in J_{1},
\end{aligned}
$$

hence, we have

$$
\begin{aligned}
& \int_{t_{i-1}}^{t_{i}}\left(\left|\dot{v}_{i}(t)\right|^{2}+\left(A(t) v_{i}(t), v_{i}(t)\right)-a_{i}\left|v_{i}(t)\right|^{2}\right) d t \geqslant c_{i}\left\|v_{i}\right\|^{2}, i \in J_{0}, \\
& \int_{t_{i-1}}^{t_{i}}\left(\left|\dot{v}_{i}^{+}(t)\right|^{2}+\left(A(t) v_{i}^{+}(t), v_{i}^{+}(t)\right)-a_{i}\left|v_{i}^{+}(t)\right|^{2}\right) d t \geqslant c_{i}^{+}\left\|v_{i}\right\|^{2}, i \in J_{1}, \\
& \int_{t_{i-1}}^{t_{i}}\left(\left|\dot{v}_{i}^{-}(t)\right|^{2}+\left(A(t) v_{i}^{-}(t), v_{i}^{-}(t)\right)-a_{i}\left|v_{i}^{-}(t)\right|^{2}\right) d t \leqslant-c_{i}^{-}\left\|v_{i}\right\|^{2}, i \in J_{1},
\end{aligned}
$$

where the constants

$$
\begin{aligned}
& c_{i}=1-\frac{\max \left\{a_{i}, 0\right\}}{\lambda_{1}^{i}}, i \in J_{0}, \\
& c_{i}^{+}=1-\frac{a_{i}}{\lambda_{n_{i}+1}^{i}}, c_{i}^{-}=\frac{a_{i}}{\lambda_{n_{i}}^{i}}-1, i \in J_{1},
\end{aligned}
$$

are all positive. Referring to the decomposition (3.4), write

$$
u_{n}=\sum_{i \in J_{0}} v_{n i}+\sum_{i \in J_{1}}\left(v_{n i}^{+}+v_{n i}^{-}\right)+w_{n}, \bar{u}_{n}=\sum_{i \in J_{0}} v_{n i}+\sum_{i \in J_{1}}\left(v_{n i}^{+}-v_{n i}^{-}\right)-w_{n} .
$$

Then, we have

$$
\begin{aligned}
\left(\varphi^{\prime}\left(u_{n}\right), \bar{u}_{n}\right)= & \sum_{i \in J_{0}} \int_{t_{i-1}}^{t_{i}}\left(\left|\dot{v}_{n i}(t)\right|^{2}-a_{i}\left|v_{n i}(t)\right|^{2}\right) d t+\sum_{i \in J_{1}} \int_{t_{i-1}}^{t_{i}}\left(\left|\dot{v}_{n i}^{+}(t)\right|^{2}-a_{i}\left|v_{n i}^{+}(t)\right|^{2}\right) d t \\
& -\sum_{i \in J_{1}} \int_{t_{i-1}}^{t_{i}}\left(\left|\dot{v}_{n i}^{-}(t)\right|^{2}-a_{i}\left|v_{n i}^{-}(t)\right|^{2}\right) d t-\int_{0}^{1}\left|\dot{w}_{n}(t)\right|^{2} d t+\sum_{i=1}^{m+1} a_{i} \int_{t_{i-1}}^{t_{i}}\left|w_{n}(t)\right|^{2} d t \\
& +2 \sum_{i \in J_{1}} a_{i} \int_{t_{i-1}}^{t_{i}}\left(v_{n i}^{-}(t), w_{n}(t)\right) d t+\int_{0}^{1}\left(A(t) w_{n}(t), w_{n}(t)\right) d t \\
& +\sum_{i \in J_{0}} \int_{t_{i-1}}^{t_{i}}\left(A(t) v_{n i}(t), v_{n i}(t)\right) d t+\sum_{i \in J_{1}} \int_{t_{i-1}}^{t_{i}}\left(A(t) v_{n i}^{+}(t), v_{n i}^{+}(t)\right) d t
\end{aligned}
$$




$$
\begin{aligned}
& -\sum_{i \in J_{1}} \int_{t_{i-1}}^{t_{i}}\left(A(t) v_{n i}^{-}(t), v_{n i}^{-}(t)\right) d t-\int_{0}^{1}\left(g\left(t, u_{n}(t)\right), \bar{u}_{n}\right) d t \\
& -\sum_{i=1}^{m}\left(I_{i}\left(w_{n}\left(t_{i}\right)\right), w_{n}\left(t_{i}\right)\right),
\end{aligned}
$$

From $\varphi^{\prime}\left(u_{n}\right) \rightarrow 0$ as $n \rightarrow \infty,(1.3),(1.4),(3.5),(3.6)$, (3.7) and (3.8), we have

$$
\begin{aligned}
& \sum_{i \in J_{0}} c_{i}\left\|v_{n i}\right\|^{2}+\sum_{i \in J_{1}}\left(c_{i}^{+}\left\|v_{n i}^{+}\right\|^{2}+c_{i}^{-}\left\|v_{n i}^{-}\right\|^{2}\right)+c_{3} \sum_{i=1}^{m}\left|w_{n}\left(t_{i}\right)\right|^{\mu} \\
& \leqslant c_{8}\left(\left\|w_{n}\right\|^{2}+\sum_{i \in J_{1}}\left\|v_{n i}^{-}\right\|\left\|w_{n}\right\|+\left\|u_{n}\right\|^{r-1}\left\|\bar{u}_{n}\right\|+\left\|\bar{u}_{n}\right\|+1\right),
\end{aligned}
$$

where $C_{8}$ is a positive constant. Since $\max _{i}\left|w\left(t_{i}\right)\right|$ defines an equivalent norm on $\mathcal{M}, \mu>2,\left\|\bar{u}_{n}\right\|=\left\|u_{n}\right\|$, and $r<2$, the boundedness of

$$
\left\|u_{n}\right\|=\sum_{i \in J_{0}}\left\|v_{n i}\right\|^{2}+\sum_{i \in J_{1}}\left(\left\|v_{n i}^{+}\right\|^{2}+\left\|v_{n i}^{-}\right\|^{2}\right)+\left\|w_{n}\right\|^{2}
$$

follows. That is $\varphi$ satisfies (PS)-condition.

Next, we will apply saddle point theorem to the splitting

$$
W=\left(\bigoplus_{i \in J_{i}} \mathcal{M}_{i}^{-} \oplus \mathcal{M}\right) \oplus\left(\bigoplus_{i \in J_{0}} \mathcal{N}_{i} \oplus \bigoplus_{i \in J_{1}} \mathcal{N}_{i}^{+}\right)=W_{1} \oplus W_{2}
$$

For $u=\sum_{i \in J_{1}} \mathcal{N}_{i}^{-}+w \in W_{1}$, from (3.2), (3.3), (3.5), (3.6) and (3.7), we have

$$
\begin{aligned}
\varphi(u) & =\frac{1}{2}\left[\sum_{i \in J_{1}} \int_{t_{i-1}}^{t_{i}}\left(\left|\dot{v}_{i}^{-}(t)\right|^{2}-a_{i}\left|v_{i}^{-}(t)\right|^{2}+\left(A(t) v_{i}^{-}(t), v_{i}^{-}(t)\right)\right) d t\right. \\
& \left.+\int_{0}^{1}(A(t) w(t), w(t)) d t+\int_{0}^{1}|\dot{w}(t)|^{2} d t-\sum_{i=1}^{m+1} a_{i} \int_{t_{i-1}}^{t_{i}}|w(t)|^{2} d t\right] \\
& -\sum_{i \in J_{1}} a_{i} \int_{t_{i-1}}^{t_{i}}\left(v_{i}^{-}(t), w(t)\right) d t-\int_{0}^{1} \mathrm{G}(t, u(t)) d t-\sum_{i=1}^{m} \hat{I}_{i}\left(w\left(t_{i}\right)\right) \\
& \leqslant-\frac{1}{2} \sum_{i \in J_{1}} c_{i}^{-}\left\|v_{i}^{-}\right\|^{2}-C_{6} \sum_{i=1}^{m}\left|w\left(t_{i}\right)\right|^{\mu}+C_{9}\left(\|w\|^{2}+\sum_{i \in J_{1}}\left\|v_{i}^{-}\right\|\|w\|+\|u\|^{r}+1\right) .
\end{aligned}
$$

Since $\max _{i}\left|\mathcal{w}\left(t_{i}\right)\right|$ defines an equivalent norm on $\mathcal{M}, \mu>2$, and $r<2$, it follows that $\varphi(u) \rightarrow-\infty$ as $\|u\|^{2}=\sum_{i \in J_{1}}\left\|v_{i}^{-}\right\|^{2}+\|w\|^{2} \rightarrow \infty$. On the other hand, for $u=\sum_{i \in J_{0}} v_{i}+\sum_{i \in J_{1}} v_{i}^{+} \in W_{2}$, from (3.2), (3.5), (3.6) and (3.7), we have

$$
\begin{aligned}
\varphi(\mathfrak{u}) & =\frac{1}{2}\left[\sum_{i \in J_{0}} \int_{t_{i-1}}^{t_{i}}\left(\left|\dot{v}_{i}(t)\right|^{2}-a_{i}\left|v_{i}(t)\right|^{2}+\left(A(t) v_{i}(t), v_{i}(t)\right)\right) d t\right. \\
& \left.+\sum_{i \in J_{1}} \int_{t_{i-1}}^{t_{i}}\left(\left|\dot{v}_{i}^{+}(t)\right|^{2}-a_{i}\left|v_{i}^{+}(t)\right|^{2}+\left(A(t) v_{i}^{+}(t), v_{i}^{+}(t)\right)\right) d t\right]-\int_{0}^{1} G(t, u(t)) d t \\
& \geqslant \frac{1}{2}\left[\sum_{i \in J_{0}} c_{i}\left\|v_{i}\right\|^{2}+\sum_{i \in J_{1}} c_{i}^{+}\left\|v_{i}^{+}\right\|^{2}\right]-C_{10}\left(\|u\|^{r}+1\right) .
\end{aligned}
$$

Since $r<2$, it follows from (3.9) that $\varphi(u)$ is bounded below on $W_{2}$. Thus, $\varphi(u)$ has a critical point by Lemma 2.1. Then problem (1.1) has at least one solution. 


\section{Acknowledgment}

This work was supported by the NNSF of China(No. 11301108, No. 61563013) and Guangxi Natural Science Foundation(No. 2016GXNSFAA380082).

\section{References}

[1] R. P. Agarwal, T. G. Bhaskar, K. Perea, Some results for impulsive problems via Morse theory, J. Math. Anal. Appl., 409 (2014), 752-759. 1

[2] D. Bainnov, P. Simeonov, Impulsive Differential Equations: Periodic Solutions and Applications, Longman Scientific Technical, Harlow, (1993). 1

[3] P. Chen, X. H. Tang, Existence of solutions for a class of $p$-Laplacian systems with impulsive effects, Taiwanese J. Math., 16 (2012), 803-828. 1

[4] A. A. Chikrii, I. I. Matychyn, K. A. Chikrii, Differential games with impulse control, Birkhuser Boston, Boston, (2007). 1

[5] E. Crück, M. Quincampoix, P. Saint-Pierre, Pursuit-evasion games with impulsive dynamics, Birkhuser Boston, Boston, (2007). 1

[6] W.-Z. Gong, Q.-F. Zhang, X. H. Tang, Existence of subharmonic solutions for a class of second-order p-Laplacian systems with impulsive effects, J. Appl. Math., 2012 (2012), 18 pages. 1

[7] S. T. Kyritsi, N. S. Papageorgiou, On superquadratic periodic systems with indefinite linear part, Nonlinear Anal., 72 (2010), 946-954. 1

[8] V. Lakshmikantham, D. D. Bănov, P. S. Simeonov, Theory of Impulsive Differential Equations, World Scientific Press, Singapore, (1989). 1

[9] J. J. Nieto, D. O'Regan, Variational approach to impulsive differential equations, Nonlinear Anal. Real World Appl., 10 (2009), 680-690. 1

[10] P. H. Rabinowitz, Minimax methods in critical point theory with applications to differential equations, Mathematical Society, Providence, (1986). 2, 2.1

[11] L. Stone, B. Shulgin, Z. Agur, Theoretical examination of the pulse vaccination policy in the SIR epidemic model, Math. Comput. Modelling, 31 (2000), 207-215. 1

[12] J. Sun, H. Chen, L. Yang, Variational methods to fourth-order impulsive differential equations, J. Appl. Math. Comput., 35 (2011), 323-340. 1

[13] Q. Wang, M. Wang, Existencce of solution for impulsive differential equations with indefinite linear part, Appl. Math. Lett., 51 (2016), 41-47. 1

[14] Q.-F. Zhang, Existence and multiplicity of fast homoclinic solutions for a class of damped vibration problems with impulsive effects, Abstr. Appl. Anal., 2014 (2014), 12 pages.

[15] X. Zhang, X. Tang, A note on the minimal periodic solutions of nonconvex super-linear Hamiltonian system, Appl. Math. Comput., 219 (2013), 7586-7590.

[16] X. Zhang, X. Tang, Non-constant periodic solutions for second order Hamiltonian system involving the p-Laplacian, Adv. Nonlinear Stud., 13 (2013), 945-964.

[17] X. Zhang, X. Tang, Some united existence results of periodic solutions for non-quadratic second order Hamiltonian systems, Commun. Pure Appl. Anal., 13 (2014), 75-95. 1 